\title{
Femtosecond laser-induced microstructures on diamond for microfluidic sensing device applications
}

\author{
Shi Su, ${ }^{1}$ Jiangling Li, ${ }^{1}$ Graham C. B. Lee, ${ }^{2}$ Kate Sugden, ${ }^{2}$ David Webb, ${ }^{2}$ and Haitao Ye ${ }^{1, a)}$ \\ ${ }^{1}$ School of Engineering and Applied Science, Aston University, Aston Triangle, Birmingham, B4 7ET, \\ United Kingdom \\ ${ }^{2}$ Aston Institute of Photonic Technologies (AIPT), Aston University, Aston Triangle, Birmingham B4 7ET, \\ United Kingdom
}

(Received 3 May 2013; accepted 31 May 2013; published online 13 June 2013)

\begin{abstract}
This paper reported a three-dimensional microfluidic channel structure, which was fabricated by Yb:YAG $1026 \mathrm{~nm}$ femtosecond laser irradiation on a single-crystalline diamond substrate. The femtosecond laser irradiation energy level was optimized at $100 \mathrm{kHz}$ repetition rate with a sub-500 femtosecond pulse duration. The morphology and topography of the microfluidic channel were characterized by a scanning electron microscope and an atomic force microscope. Raman spectroscopy indicated that the irradiated area was covered by graphitic materials. By comparing the cross-sectional profiles before/after removing the graphitic materials, it could be deduced that the microfluidic channel has an average depth of $\sim 410 \mathrm{~nm}$ with periodical ripples perpendicular to the irradiation direction. This work proves the feasibility of using ultra-fast laser inscription technology to fabricate microfluidic channels on biocompatible diamond substrates, which offers a great potential for biomedical sensing applications. (C) 2013 AIP Publishing LLC. [http://dx.doi.org/10.1063/1.4811170]
\end{abstract}

Single-crystalline diamond films offer some extremely remarkable properties, such as the highest thermal conductivity $\left(2 \times 10^{3} \mathrm{~W} \mathrm{~m}^{-1} \mathrm{~K}^{-1}\right)$, high mechanical hardness ( $\sim 100 \mathrm{GPa})$, wide bandgap $(5.47 \mathrm{eV})$, high electric breakdown field $\left(\sim 2 \times 10^{7} \mathrm{~V} \mathrm{~cm}^{-1}\right)$, high carrier mobility (2400 $\mathrm{cm}^{2} \mathrm{~V}^{-1} \mathrm{~s}^{-1}$ for electrons, $2100 \mathrm{~cm}^{2} \mathrm{~V}^{-1} \mathrm{~s}^{-1}$ for holes), and chemical inertness, as well as outstanding biocompatibility. ${ }^{1}$ The diamond films therefore have been utilized for substrate materials of power devices, ${ }^{2}$ cantilever scanning probes, ${ }^{3}$ microelectro-mechanical system (MEMS), ${ }^{4}$ and biological/ electrochemical electrodes, ${ }^{5}$ etc. However, little progress on in vivo biocompatible devices has been reported, for the extreme hardness of diamond increases the difficulties in fabrication of nano/micrometer structures on its surface. ${ }^{6}$ Previously, surface modification on diamond substrates was performed by focused ion beam (FIB) technology, but it required a longer time (up to hours) to complete the entire fabrication process. ${ }^{7}$ Reactive ion etching (RIE) has been offered as an alternative method with an inefficient etching rate of $\sim 10 \mu \mathrm{m} \mathrm{h}^{-1}$ on the diamond surface. ${ }^{8}$ For FIB and RIE techniques, both the harsh environmental requirement and diamond crystal lattices damage have limited the practical applications in addition to the unsatisfactory etching efficiency.

Recently, the femtosecond (fs) laser technology has attracted great interest in scientific and manufacturing communities for its precision and damage-free fabrication capability. ${ }^{9}$ The fs-laser technology has enabled microstructuring of different materials, including metals, dielectrics, as well as semiconductors, in terms of applications in photonics, devices. ${ }^{10}$ Furthermore, a number of research groups have carried out fs-laser inscription on diamond/ diamond-like carbon materials. Ozkan et al. investigated the

\footnotetext{
${ }^{\text {a) }}$ Author to whom correspondence should be addressed. Electronic mail: h.ye@aston.ac.uk
}

periodical ripple patterns observed on the sample surface after fs-laser irradiation and attributed it as being caused by light reflection from surface and the laser-generated plasma. ${ }^{11}$ Shinoda et al. applied the fs-laser technology to fabricate $\sim 300 \mu \mathrm{m}$ long parallelepiped pillar structures on single-crystalline diamond. ${ }^{12}$ Jeschke et al. proposed a nonequilibrium model for transition from diamond to graphitized composition induced by the fs-laser. ${ }^{13}$ However, few results related to near-infrared fs-laser inscription on diamond substrates have been reported. In this paper, we report the fundamental properties of the fs-laser inscribed microstructures that we intends to use in the future as the basis for microfluidic sensing devices.

The experimental setup of the fs-laser equipment is shown in Figure 1. Sub-500 fs-laser pulses were generated by a mode-locked Yb:YAG laser system with a center wavelength of $1026 \mathrm{~nm}$ (Amplitude System s-Pulse HP) and a repetition rate of $100 \mathrm{kHz}$. Inscription work was carried out under a $20 \times$ objective (Mititoyo MPlan Apo NIR Series) with a numerical aperture of 0.4. The effective laser spot size had an approximate diameter of $2 \mu \mathrm{m}$. The fs-laser power was set at $17.5 \mathrm{~nJ}$ per pulse. This threshold irradiation energy power was lower than a previously reported laser power threshold $(72 \mathrm{~nJ}$ at $800 \mathrm{~nm}) .{ }^{12}$ The single-crystalline diamond was secured to a sub-nanometer precision XY airbearing stage with a mechanical Z-translation (ABL1000, Aerotech) system. The motion of translation stages was controlled through a custom written CNC program. A piece of polished single-crystalline diamond film with a size of $3 \times 3$ $\mathrm{mm}^{2}$ and $0.7 \mathrm{~mm}$ thick (Element Six Ltd.) was used as the substrate in this experiment. Before the fs-laser irradiation, the diamond was subjected to the process of removing surface contaminations. ${ }^{6}$ After finishing surface cleaning process, the diamond substrate was fixed on a flat glass slide for insertion into the fs-inscription system. After fs-laser irradiation, the diamond substrate was characterized by SEM, 


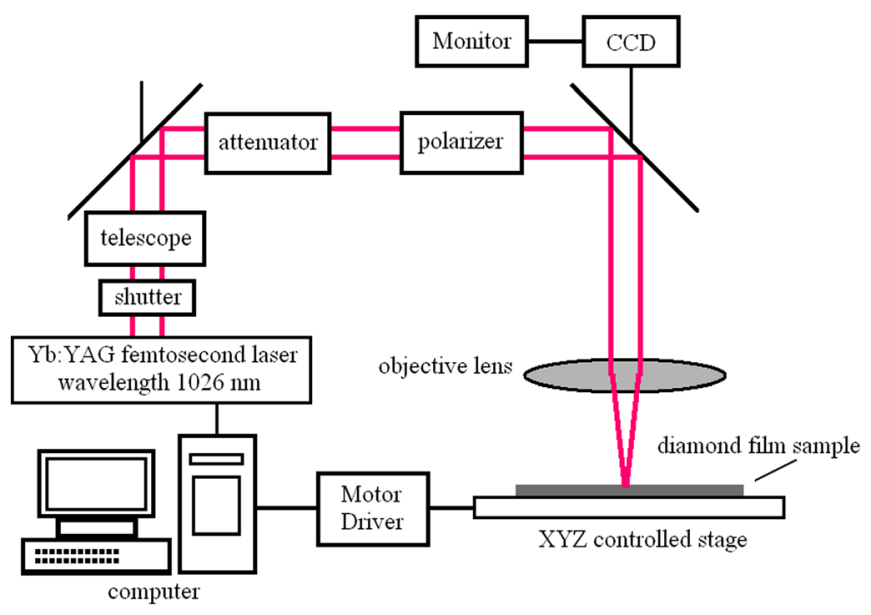

FIG. 1. Schematic illustration of experimental setup of femtosecond laser system.

Raman spectroscopy and AFM, after being subjected to the following further cleaning process: (1) immersion in acetone/isopropanol ultrasonic bath (room temperature, $30 \mathrm{~min} /$ $30 \mathrm{~min}$ ) to remove organic contamination; (2) boiling in aqua regia $\left(70^{\circ} \mathrm{C}, 2 \mathrm{~h}\right)$ to remove metallic contamination; (3) boiling in $95 \% \mathrm{H}_{2} \mathrm{SO}_{4}+36 \% \mathrm{HNO}_{3}\left(9: 1,100{ }^{\circ} \mathrm{C}, 8 \mathrm{~h}\right)$ to partially remove the graphitic layer generated by the fslaser. ${ }^{13,14}$ Finally, in order to fully remove the residue of the graphitic material, the diamond substrate was subjected to hydrogen-plasma etching process was performed using a microwave plasma enhanced chemical vapor deposition system (MPECVD, Seki Technotron Corp. ASTeX 5010). The hydrogen plasma was ignited by a $2.45 \mathrm{GHz}$ microwave generator at $900 \mathrm{~W}$ and maintained for $6 \mathrm{~h}$, with a diluted hydrogen gas flow rate of $200 \mathrm{sccm}$ under 50 Torr pressure. A Raman spectrometer (Nicolet Almega XR dispersive type, $785 \mathrm{~nm}$, Thermo Ltd.) equipped with an optical microscope (Olympus TE-Si CCD) was used to observe and locate the graphitic area. The topography of the fs-laser induced pattern was characterized by a contact mode AFM (Autoprobe M5, Park Scientific Instrument) with a scanning area $100 \times 100 \mu \mathrm{m}^{2}$.

The whole topography of the fs-laser inscribed pattern is shown in Figure 2(a). A zigzag microfluidic structure is clearly observed with a size of $\sim 200 \times 200 \mu \mathrm{m}^{2}$. It can be seen that the texture of the channel shows a well-defined shape as expected. Figure 2(b) shows the magnified SEM image at the edge of the fs-laser inscribed channels as labeled with red square in Figure 2(a). It is obvious that this part of the channel near a corner shows a very rough and porous 'lava-like' morphology, which consists of hollowed and crystalline materials. During the inscription process, the fslaser had been focusing on the irradiated channels for a relatively longer time at the corner of the channel. Therefore, this area experienced excessive exposure to the fs laser irradiation and more fragmental diamond crystals were exposed. Figure 2(c) shows the magnified SEM image at the center of the fs-laser inscribed channels as labeled with the blue square in Figure 2(a). It shows a less porous, but more uniform morphology, compared with the image at the corner shown in Figure 2(b). Figure 2(d) shows the SEM image at the center of the fs-laser inscribed channels after fully removing the surface graphitic materials. It can be seen that the channels' surface morphology becomes smoother and periodical ripples are revealed in comparison with Figures 2(b) and $2(\mathrm{c})$.
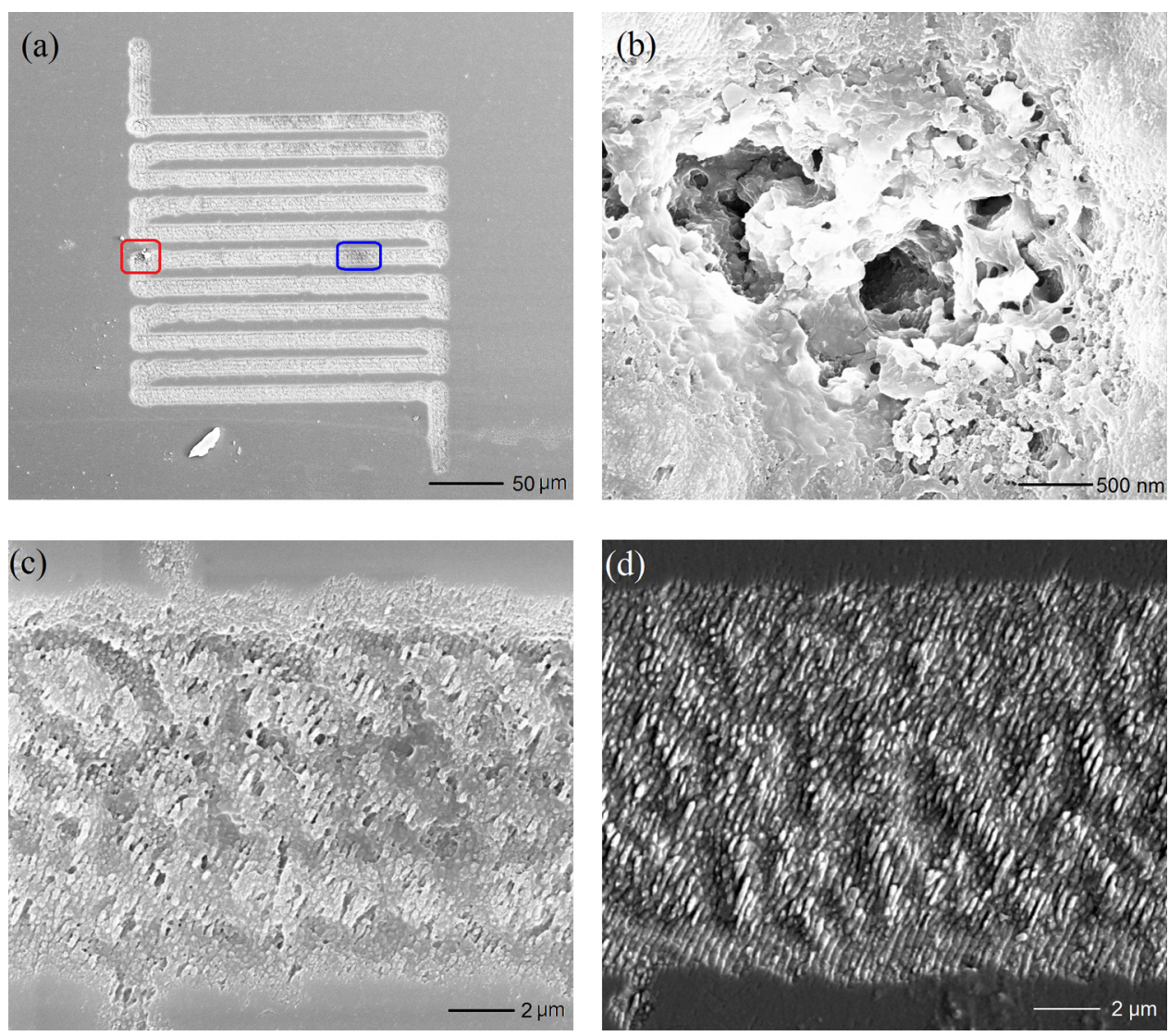

FIG. 2. (a) Topographic SEM image of fs-laser inscribed microfluidic channel pattern on a diamond substrate; (b) high magnification porous structure at corner of the fs-laser inscribed pattern (corresponding to red square area in Fig. 2(a)); and high magnification SEM image of the fs-laser inscribed channel (corresponding to blue square area in Fig. 2(a)): (c) before removing graphitic layer; (d) after removing graphitic layer. 
For laser pulses irradiating dielectric materials at the picosecond-level or even longer, the heating mechanism can be explained as an increase in the kinetic energy of conduction band electrons followed by transfer of energy to the crystal lattice. This thermal dissipation will influence the surrounding lattices and finally reach a thermal equilibrium with them. In comparison, for femtosecond-level irradiation, the photon energy transfers from free electrons to the lattice within only hundreds of femtosecond, which is much shorter than the free time of diamond atomic lattice collisions $\left(10^{-11} \sim 10^{-13} \mathrm{~s}\right) .{ }^{15}$ As the fs-laser photon energy $(1.21 \mathrm{eV})$ is higher than the reconstruction energy of diamond surface $\pi$-bonds $(\sim 0.68 \mathrm{eV})$, the laser pulse energy will lead to multiphoton absorption and rapid ionization of the surrounding materials to create a dense electron plasma. ${ }^{16}$ Thus, there is no thermal equilibrium between the lattice and electrons, while the phase transformation is driven by hot-electron plasma induced by the fs-laser pulses. The surrounding diamond materials were maintained stable due to high thermal dissipation, whilst the fs-laser irradiated area reached a high temperature sufficient for the phase transformation. ${ }^{17}$

After removing the surface graphitic layer, the ripples in the channels can be clearly observed and the ripple fringes have a periodical repetition. Shinoda and Bhardwaj, et al. suggested that the spatial period fringe $\Lambda$ can be deduced by ${ }^{12,18}$

$$
\Lambda=\lambda / 2 n,
$$

where $\lambda$ is the induced fs-laser free space wavelength, and $n$ is the refractive index. Furthermore, the refractive index $n$ can be calculated by Sellmeier equation as

$$
n^{2}=1+\frac{A_{1} \lambda^{2}}{\lambda^{2}-\lambda_{1}^{2}}+\frac{A_{2} \lambda^{2}}{\lambda^{2}-\lambda_{2}^{2}},
$$

where $A_{1}, A_{2}, \lambda_{1}$, and $\lambda_{2}$ are Sellmeier coefficients. In our experiment, $\lambda$ is $1026 \mathrm{~nm}$ and Sellmeier coefficients of diamond materials are: $A_{1}=0.3306, A_{2}=4.3356, \lambda_{1}=0.175 \mu \mathrm{m}$, and $\lambda_{2}=0.106 \mu \mathrm{m} .{ }^{19}$ Thus, the corresponding refractive index can be deduced as $n=2.39$. From Eq. (1), one can calculate that the theoretical value of the spatial period fringe $\Lambda$ is around $215 \mathrm{~nm}$. In the SEM image in Figure 2(d), the spatial period observed for our sample is in a good agreement with the theoretical prediction.

The fs-laser irradiated graphitic regions were then characterized by Raman spectroscopy, as shown in Figure 3 . Curve (a) shows the Raman spectra from the diamond substrate before fs-laser inscription. A sharp peak at $1332 \mathrm{~cm}^{-1}$, which is derived from the $T_{2 \mathrm{~g}}$ zone center mode, indicates the substrate is fully composed by carbon $s p^{3}$ bonds. Curve (b) demonstrates the Raman spectrum obtained from the focused area as indicated in Figure 2(b). For curve (b), there are four characteristic Raman peaks, which are located at $1600 \mathrm{~cm}^{-1}, 1337 \mathrm{~cm}^{-1}, 1243 \mathrm{~cm}^{-1}$, and $1190 \mathrm{~cm}^{-1}$. The peak located at $\sim 1600 \mathrm{~cm}^{-1}$ can be attributed to the $G$ peak, which is always assigned to carbon zone center photons of $E_{2 \mathrm{~g}}$ symmetry. ${ }^{20}$ The other three Raman peaks are due to the presence of inhomogeneous micro stress on the diamond grains. ${ }^{21}$ The peak at $1337 \mathrm{~cm}^{-1}$ corresponds to the diamond

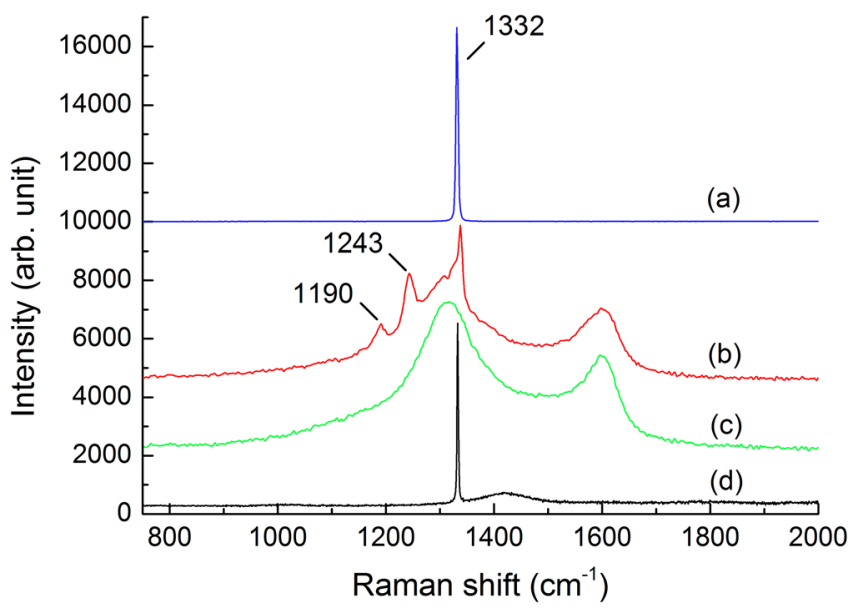

FIG. 3. The Raman spectra of curve (a): the diamond substrate without fslaser irradiation; curve (b): the porous structure at the corner of the fs-laser inscribed pattern (corresponding to Fig. 2(b)); curve (c): the fs-laser inscribed channel groove before removing graphitic layer (corresponding to Fig. 2(c)); curve (d): the fs-laser inscribed channel groove after removing graphitic layer (corresponding to Fig. 2(d)).

feature peak. The peak at $1190 \mathrm{~cm}^{-1}$ might be attributed to either the CC interring stretch vibration or $\nu_{1}$ mode of transpolyacetylene (trans-PA). ${ }^{22}$ The latter was derived from the sum and combinations of $\mathrm{C}=\mathrm{C}$ chain stretching and $\mathrm{C}-\mathrm{H}$ wagging modes. The sharp peak at $1243 \mathrm{~cm}^{-1}$ was another evidence of the existence of trans-PA on the surface. ${ }^{23}$ The Raman spectra indicate this area is $s p^{3}$ bonding dominant. From the SEM image shown in Figure 2(b), it can be seen that this area is constructed of hollowed and crystalline materials. It was assumed that the structure was formed because of the motor stage was changing direction at the corner of the channels and so the beam focused on the corner for a relatively longer time than elsewhere in the channels. Therefore, this area experienced excessive irradiation and more fragmental diamond crystals were exposed.

Curves (c) and (d) in Figure 3 correspond to the fs-laser inscribed area in the channels before/after removing the graphitic layer, as shown in Figure 2(c) and 2(d), respectively. The curve (c) has two wide shoulders located at $\sim 1320 \mathrm{~cm}^{-1}$ ( $D$ peak), which is derived from the $K$-point photons from the breathing mode of $A_{1 \mathrm{~g}}$ symmetry, and $\sim 1600 \mathrm{~cm}^{-1}$ ( $G$ peak). ${ }^{20}$ After fitting the Raman spectra (c), the $D$ peak was found at $1317 \mathrm{~cm}^{-1}$ with a FWHM of $3.8 \mathrm{~cm}^{-1}$. The intensity ratio of $I(D) / I(G)$ was determined as $\sim 1.8$, which suggested that the area might be covered by nano-crystalline graphite. ${ }^{24}$ This result implied that the fs-laser irradiated the diamond surface and formed a relatively uniform graphitic layer. The Raman spectroscopy confirmed the graphitization layer on the fs-laser irradiated area. After the surface graphitic layer was removed, the Raman spectra (d) show a diamond characteristic peak at $1332 \mathrm{~cm}^{-1}$ and a minor shoulder at $\sim 1450 \mathrm{~cm}^{-1}$. The $1450 \mathrm{~cm}^{-1}$ minor shoulder can be associated with the $\nu_{3}$ modes of transpolyacetylene. ${ }^{25}$ The disappearance of peak $D$ and $G$, as well as the re-appearance of diamond characteristic peak proved that the surface graphitic layer was fully removed by our sulfuric acid/hydrogen plasma treatment.

The AFM measurements were performed before and after the surface graphitic layer removed, and results were 
(a)

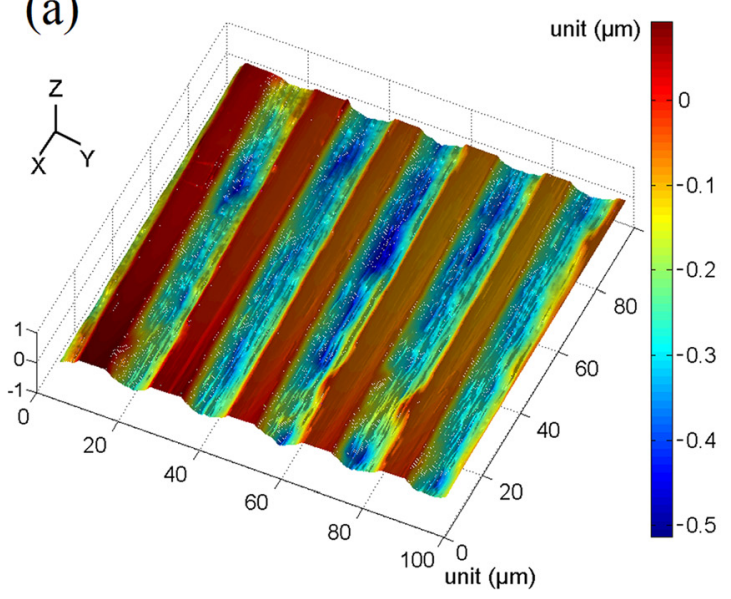

(b)

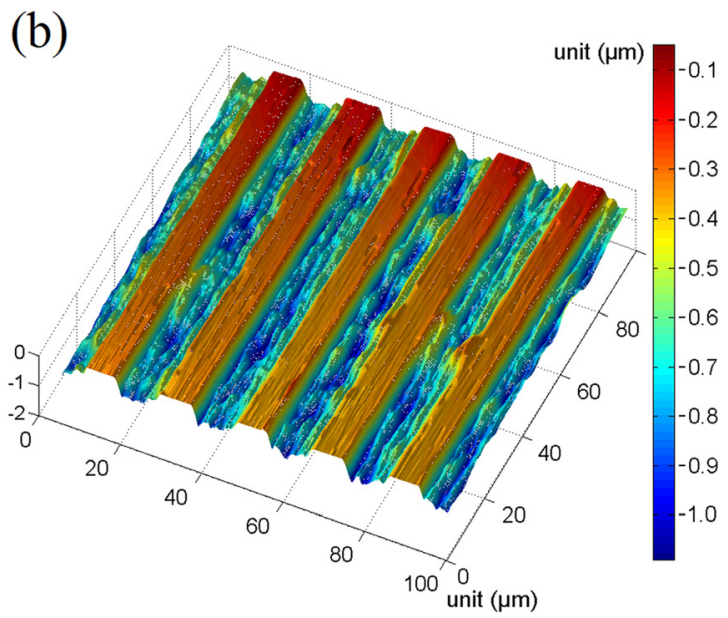

FIG. 4. AFM image of fs-laser irradiated area on diamond substrate: (a) before removing graphitic layer; and (b) after removing graphitic layer. The XY axis corresponds to the surface of diamond substrate, while the $\mathrm{Z}$ axis is the depth on its surface.

shown in Figure 4. A reference surface roughness measurement was obtained by scanning a $30 \times 30 \mu \mathrm{m}^{2}$ area on an uninscribed area. The root mean square (RMS) roughness of the substrate without fs-laser irradiation was $4.9 \mathrm{~nm} .^{6}$ In comparison, the RMS roughness of irradiated area was $64.3 \pm 10.8 \mathrm{~nm}$ in the microfluidic channels. The increased roughness was due to the formation of the graphitic clusters by the laser-induced plasma. As the graphitic layer was removed, the RMS further increased up to $143.0 \pm 11.7 \mathrm{~nm}$ in the channels. This could be explained by the exposure of the ripples and fringes after the graphitic clusters were fully removed. The cross-sectional profiles of microfluidic channels were measured and plotted in Figure 5. The mean value of maximum depth (RTM) in the microfluidic channels was $\sim 245 \mathrm{~nm}$, which could be obtained from Figure 5(a) and the RTM increased up to $\sim 410 \mathrm{~nm}$ after the graphitic layer was removed. Comparing the difference between the statistic average depth of the valleys in Figure 5 before and after removing surface graphitic layer, it can be deduced that the graphitic layer has a thickness of $\sim 165 \mathrm{~nm}$. The graphitic layers generated by the fs-laser irradiation not only covered the channels, but also accumulated partially on the edges of the channels where no inscription occurred. This phenomenon was consistent with the results from Okuchi et $a l .{ }^{26}$ In Figure 5(b), it can be seen that the cross-sectional profile of the microfluidic channel was asymmetric. Other shoulder peaks were observed to be associated with each valley peak. Similar results were reported in the fs-laser inscription on fused silica. ${ }^{27}$ This could be explained as the existence of mirrors in the optical path, or the tilted attachment between diamond substrate and the glass slide. Either of the above factors will result in a tilted plasma dot in the diamond substrates, which leads to an asymmetric inscription. However, these asymmetric structures can be tuned and controlled by the optimization of the process.

In summary, we have demonstrated that femtosecond laser-induced microfluidic channels can be fabricated on single-crystalline diamonds. The morphology of the fs-laser inscribed area was also revealed as well. The fs-laser inscription technique offers a faster and more convenient approach to develop 3D devices and structures on diamond substrates, when compared with conventional techniques such as FIB and RIE, etc. Future work will emphasize on the characterization of these devices for biomedical sensing and the theoretical modeling of the inscription process, especially with the laser focused inside the bulk of the diamond substrates.
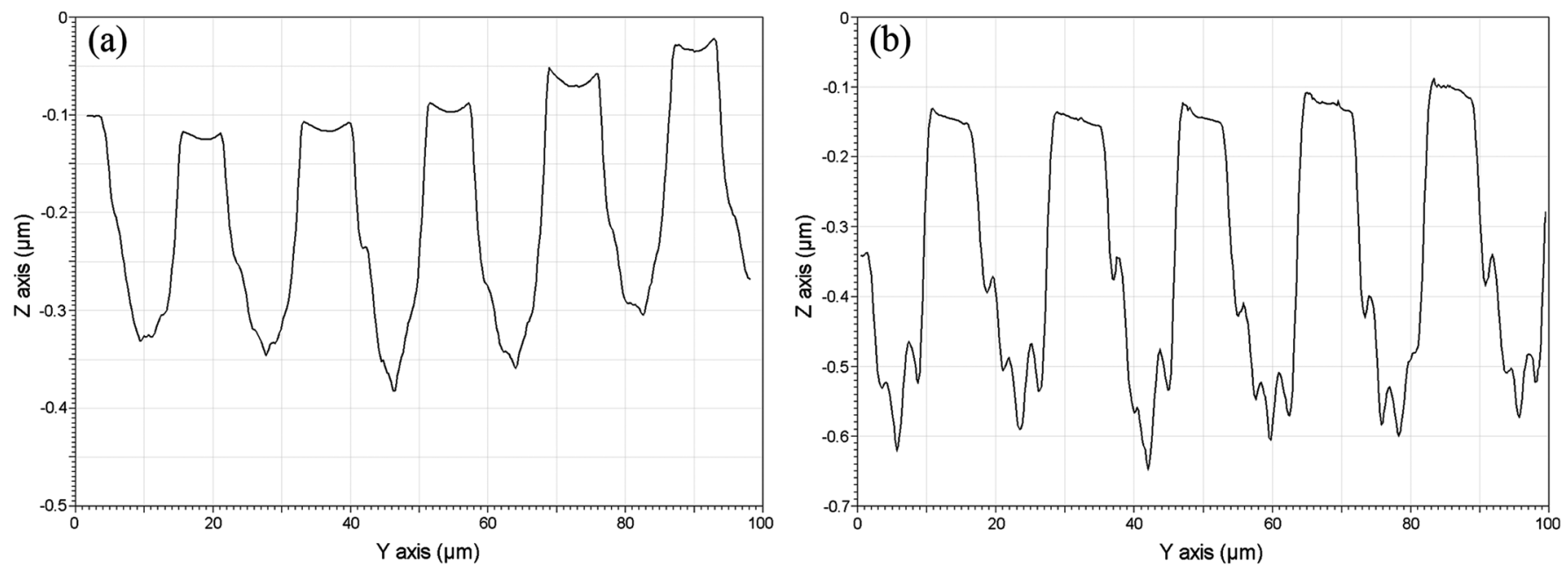

FIG. 5. Cross-section views extracted from the AFM results of microfluidic channels: (a) before removing the graphitic layer; and (b) after removing graphitic layer. A graphitic layer thickness of $\sim 165 \mathrm{~nm}$ can be deduced by comparing the relative average lowest depths of the valleys. 
This could offer a potential for the fabrication of selfembedded devices and nanostructures for a number of advanced sensing applications.

The authors want to acknowledge the funding for this study provided by the Engineering and Physical Sciences Research Council (EPSRC: EP/H034269/1).

${ }^{1}$ J. J. Gracio, Q. H. Fan, and J. C. Madaleno, J. Phys. D: Appl. Phys. 43, 374017 (2010).

${ }^{2}$ A. Denisenko and E. Kohn, Diamond Relat. Mater. 14, 491 (2005).

${ }^{3}$ E. Oesterschulze, A. Malavé, U. F. Keyser, M. Paesler, and R. J. Haug, Diamond Relat. Mater. 11, 667 (2002).

${ }^{4}$ N. Sepulveda, D. Aslam, and J. P. Sullivan, Diamond Relat. Mater. 15, 398 (2006).

${ }^{5}$ J. Iniesta, P. A. Michaud, M. Panizza, G. Cerisola, A. Aldaz, and Ch. Comninellis, Electrochim. Acta. 46, 3573 (2001).

${ }^{6}$ G. C. B. Lee, S. Su, J. Li, K. Sugden, N. Roohpour, H. Yan, and H. Ye, J. Exp. Nanosci. 7, 662 (2012).

${ }^{7}$ A. Datta, Yuh-Renn Wu, and Y. L. Wang, Phys. Rev. B 63, 125407 (2001).

${ }^{8}$ Y. Ando, Y. Nishibayashi, K. Kobashi, T. Hirao, and K. Oura, Diamond Relat. Mater. 11, 824 (2002).

${ }^{9}$ T. V. Kononenko, M. S. Komlenok, V. P. Pashinin, S. M. Pimenov, V. I. Konov, M. Neff, V. Romano, and W. Lüthy, Diamond. Relat. Mater. 18 , 196 (2009).

${ }^{10}$ G. D. Valle, R. Osellame, and P. Laporta, J. Opt. A: Pure Appl. Opt. 11, 013001 (2009).
${ }^{11}$ A. M. Ozkan, A. P. Malshe, T. A. Railkar, W. D. Brown, M. D. Shirk, and P. A. Molian, Appl. Phys. Lett. 75, 3716 (1999).

${ }^{12}$ M. Shinoda, R. R. Gattass, and E. Mazur, J. Appl. Phys. 105, 053102 (2009).

${ }^{13}$ H. O. Jeschke, M. E. Garcia, and K. H. Bennemann, Phys. Rev. B 60, R3701 (1999).

${ }^{14}$ K. E. Carr, Carbon 8, 155 (1970).

${ }^{15}$ C. Z. Wang, K. M. Ho, M. D. Shirk, and P. A. Molian, Phys. Rev. Lett. 85, 4092 (2000).

${ }^{16}$ B. C. Stuart, M. D. Feit, A. M. Rubenchik, B. W. Shore, and M. D. Perry, Phys. Rev. Lett. 74, 2248 (1995).

${ }^{17}$ M. D. Shirk, P. A. Molian, and A. P. Malshe, J. Laser Appl. 10, 64 (1998).

${ }^{18}$ V. R. Bhardwaj, E. Simova, P. P. Rajeev, C. Hnatovsky, R. S. Taylor, D. M. Rayner, and P. B. Corkum, Phys. Rev. Lett. 96, 057404 (2006).

${ }^{19}$ Optical Properties of Condensed Matter and Applications, edited by J. Singh (Wiley, Chichester, 2006), Vol. 6.

${ }^{20}$ A. C. Ferrari and J. Robertson, Phys. Rev. B. 63, 121405 (2001).

${ }^{21}$ Y. von Kaenel, J. Stiegler, J. Michler, and E. Blank, J. Appl. Phys. 81, 1726 (1997)

${ }^{22}$ Q. G. Zeng, Z. J. Ding, X. D. Tang, and Z. M. Zhang, J. Lumin. 115, 32 (2005).

${ }^{23}$ R. Pfeiffer, H. Kuzmany, P. Knoll, S. Bokova, N. Salk, and B. Günther, Diamond Relat. Mater. 12, 268 (2003).

${ }^{24}$ A. C. Ferrari and J. Robertson, Phys. Rev. B 61, 14095 (2000).

${ }^{25}$ A. C. Ferrari and J. Robertson, Philos. Trans. R. Soc. London A 362, 2477 (2004).

${ }^{26}$ T. Okuchi, H. Ohfuji, S. Odake, H. Kagi, S. Nagamoto, M. Sugata, and H. Sumiya, Appl. Phys. A 96, 833 (2009).

${ }^{27}$ M. Dubov, I. Bennion, D. N. Nikogosyan, P. Bolger, and A. V. Zayats, J. Opt. A: Pure Appl. Opt. 10, 025305 (2008). 